association value (a) in paired-associate syllable learning. Psychological Reports, $1957,3,441-452$.

SOLSO, R. L. Functional stimulus selection as related to color versus verbal stimuli. Joumal of Experimental Psychology, $1968,78,382-387$

THORNDIKE, E. L., \& LORGE. I. The teacher's word book of 30,000 words. New York: Bureau of Publications, Teachers College, Columbia University, 1944.

UNDERWOOD, B. J., HAM, M., \& EKSTRAND, B. Cue selection in paired-associate learning. Journal of Experimental Psychology, 1962, 64, 405-409.
VANDERPLAS, J. M \& GARVIN, E, Complexity, association value, and practice as factors in shape recognition following paired-associate training. Journal of Experimental Psychology, $1959,57,155-163$.

WTMER, $L$. R. The association value of three-place consonant syllables. Joumal of Genetic Psychology, 1935, 47 . 337-360.

* Part of this paper was presented at the Midwestern Psychological Association Meeting, May 1967, Chicago, Illinois.

\section{Paragraph complexity, arousal, and subjective evaluations of attractiveness*}

\author{
DAVID R. EVANS $\dagger$ \\ University of Calgary, Calgary 44, Alta., Canada
}

The purpose of this study was to test various postulates concerning the interrelationships between the arousal potential, the arousal level, and the attractiveness of a set of paragraphs differing in complexity. The effect of paragraph complexity on arousal level and ratings of attractiveness was significant. A significant U-shaped relationship between paragraph complexity and arousal level was observed. A significant negative linear relationship between paragraph arousal level and ratings of attractiveness was found. There was a significant inverted $U$-shaped relationship between paragraph complexity and ratings of attractiveness.

Berlyne (1963) has made the following postulates concerning the interrelationships between the arousal potential, arousal level, and attractiveness of a set of stimulus situations varying in arousal potential: (1) there is a U-shaped relationship between arousal level and arousal potential; (2) there is a negative linear relationship between attractiveness and arousal level; and (3) there is an inverted U-shaped relationship between attractiveness and arousal potential. The purpose of this investigation was to test Berlyne's (1963) postulates concerning the relationship between attractiveness and arousal level and between attractiveness and arousal potential. In a previous experiment, reported elsewhere (Evans, 1970a), a significant curvilinear relationship was found between arousal level and paragraph

*This study is based on a doctoral dissertation submitted in partial fulfillment of the requirements for the PhD degree, Department of Applied Psychology, The Ontario Institute for Studies in Eduation, University of Toronto. The author is indebted to F. J. Clifford, the principal of Peterborough Teachers' College, and the students of the College, who acted as Ss. and to H. I. Day, H. G. Narrol, and G. L. McDiarmid for helpful comments regarding the preparation of this report.

+Present address: Department of Educational Psychology, University of Calgary, Calgary 44, Alta.. Canada. complexity. Stimulation from sources other than paragraph complexity were assumed to vary at random among Ss; therefore, the only stimuli contributing to arousal potential systematically were the paragraphs. Thus, the observed U-shaped relationship between paragraph complexity and arousal level supports Berlyne's (1963) postulate concerning the relationship between arousal potential and arousal level. Berlyne's second postulate would suggest a negative linear relationship between paragraph arousal levels and indices of stimulus attractiveness. Finally, according to Berlyne's third postulate, there should be an inverted U-shaped relationship between paragraph complexity and stimulus attractiveness.

An attractive stimulus situation may be considered to be interesting, pleasing, and preferred. Therefore, on the basis of Berlyne's postulate, subjective evaluations of interestingness, pleasingness, and preference should decrease linearly over paragraph arousal level. Subjective evaluations of interestingness, pleasingness, and preference should also describe an inverted U-shaped function over paragraph complexity. SUBJECTS

The Ss for this study were 30 students at Peterborough Teachers' College. There were 6 males and 24 females, and each sex group had a median age of 20 years.

\section{MATERIAL}

Six paragraphs between 95 and 105 words long were used to represent differing levels of complexity. The paragraphs and the procedure by which they were selected has been given previously (Evans, 1970b). Ss were assigned randomly to rate the paragraphs in one of six orders (Evans, $1970 \mathrm{~b})$ such that five $S$ s rated the paragraphs in each order. Five sets of the six paragraphs, typed with double spacing on $8 \frac{1}{2} \times 11$ in. white paper, were collated in each order. Semantic differential forms, including the semantic scales used by Day (1967), along with two additional dimensions, "s i m ple-complex", and "likable-dislikable,", were mimeographed on $81 / 2 \times 11$ in. white paper and collated in sets of six.

\section{PROCEDURE}

The $30 \mathrm{Ss}$ were seated at separate desks in a classroom. Each $\mathrm{S}$ was given one of the sets of six paragraphs and a set of semantic differential forms. Ss were asked to write their name, age, sex, and the first word of the first paragraph in their set of paragraphs on the face sheet of the semantic differential forms. They were asked to read each paragraph and to rate each on the 21 semantic differential dimensions before proceeding to the next paragraph.

\section{RESULTS}

In a previous experiment (Evans, $1970 \mathrm{a})$, the effect of paragraph complexity on arousal level was investigated. The relationship between median GSR amplitude and paragraph complexity is shown in Fig. 1. The effect of paragraph complexity on mean GSR amplitude was significant, and a nonparametric trend analysis indicated that only the curvilinear relationship was significant. Figure 1 shows that the relationship is U-shaped in form.

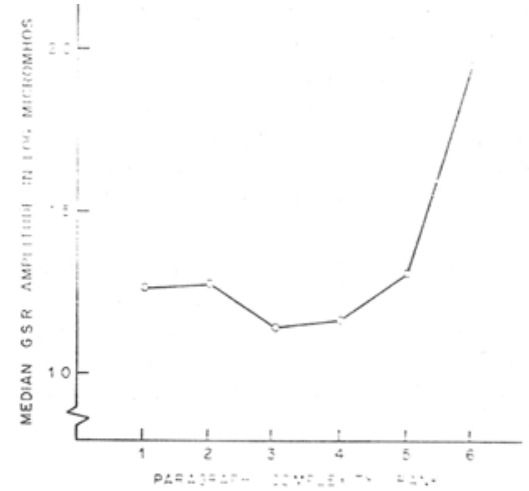

Fig. 1. Paragraph complexity and GSR a mplitude (Evans, 1970a, p. 252 ). 
Table 1

Trend Analysis for the Relationship Between Paragraph Complexity and Atractiveness Ratings

\begin{tabular}{lcc}
\hline \multicolumn{1}{c}{ Rating } & $\begin{array}{c}\text { F Ratio of } \\
\text { Linear } \\
\text { Component }\end{array}$ & $\begin{array}{c}\text { F Ratio of } \\
\text { Quadratic } \\
\text { Component }\end{array}$ \\
\hline Pleasing-Dipleating & $24.51^{*}$ & $13.40^{*}$ \\
Interesting-Boring & $20.04^{*}$ & $21.83^{*}$ \\
Lilreable-Dislikeable & $24.01^{*}$ & $32.88^{*}$ \\
\hline
\end{tabular}

tDegrees of freedom are 1 and $115 ; * p<.01$.
Table 2

Trend Analysis for the Relationship Between Paracraph Arousal Level and Attractiveness Ratings

\begin{tabular}{lcc}
\hline \multicolumn{1}{c}{ Rating } & $\begin{array}{c}\text { F Ratio of } \\
\text { Linear } \\
\text { Component }\end{array}$ & $\begin{array}{c}\text { F Ratio of } \\
\text { Quadratic } \\
\text { Component }\end{array}$ \\
\hline Pleasing-Displeasing & $38.15^{*}$ & 1.14 \\
Interesting-Boring & $43.98^{*}$ & 0.45 \\
Likeable-Dislikeable & $65.15^{*}$ & 1.38 \\
\hline
\end{tabular}

+ Degrees of freedom are 1 and $115 ; * p<.01$.

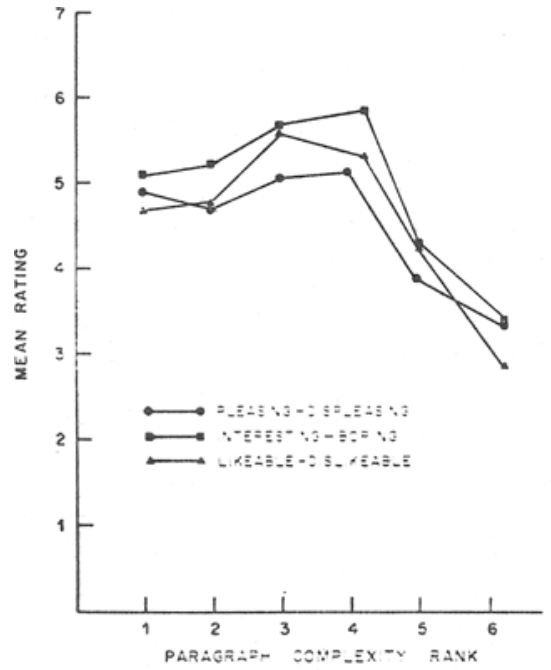

Fig. 2. Paragraph complexity and mean ratings of pleasingness, interestingness, and preference.

In the present experiment, the ratings of interestingness, pleasingness, and preference (like-dislike) for each $\mathbf{S}$ on each paragraph were tabled. The relationships between mean ratings for each of the dimensions and paragraph complexity are shown in Fig. 2. The effect of paragraph complexity on ratings of pleasingness was significant $[F(5,115)=8.50, p<.01]$. The effect of paragraph complexity on ratings of interestingness was also significant $[F(5,115)=13.00, p<.01]$.

Trend analysis indicated that both the linear and the quadratic components for each set of ratings was significant (see Table 1).

The relationship between mean ratings for each of the dimensions and paragraph arousal level is shown in Fig. 3. Trend analysis was performed on the data in this order, with orthogonal coefficients for unequal intervals calculated as suggested by Grandage (1958). Trend analysis indicates that only the linear component of the trend is significant (see Table 2).

\section{DISCUSSION}

As indicated in the introduction, the results of a previous experiment (Evans, 1970a) indicated that the relationship between paragraph complexity and arousal level is U-shaped in form. In the present experiment, the effect of paragraph complexity on ratings of pleasingness, interestingness and preference (like-dislike) was significant. Both the linear and quadratic components of the trend analysis of the relationship between paragraph complexity and attractiveness ratings were significant. This finding suggests that the relationship is best described by a quadratic function and is, therefore, curvilinear in nature. Figure 2 suggests that there is, indeed, a significant inverted U-shaped relationship between paragraph complexity and attractiveness ratings. This finding supports Berlyne's (1963) postulate that there is an inverted U-shaped relationship between arousal potential and attractiveness. The inverted U-shaped relationship between paragraph complexity and attractiveness is also in agreement with Berlyne's (1967) later hypothesis that moderate increases in arousal are rewarding (attractive), whereas extreme increases are aversive. The present findings are also in agreement with those of Kammann (1966), who found an inverted U-shaped relationship between poem complexity and poem preference.

Trend analysis of the ratings of attractiveness when paragraphs weie recorded on the basis of paragraph arousal level indicated that only the linear components of the trend were significant. Insnection of Fig. 3 indicates that there is a decreasing linear relationship between ratings of attractiveness and paragraph arousal level. This finding is in agreement with Berlyne's (1963) postulate that there is a negative linear relationship between attractiveness and arousal level and is in agreement with Berlyne's (1967) discussion concerning the interaction between arousal

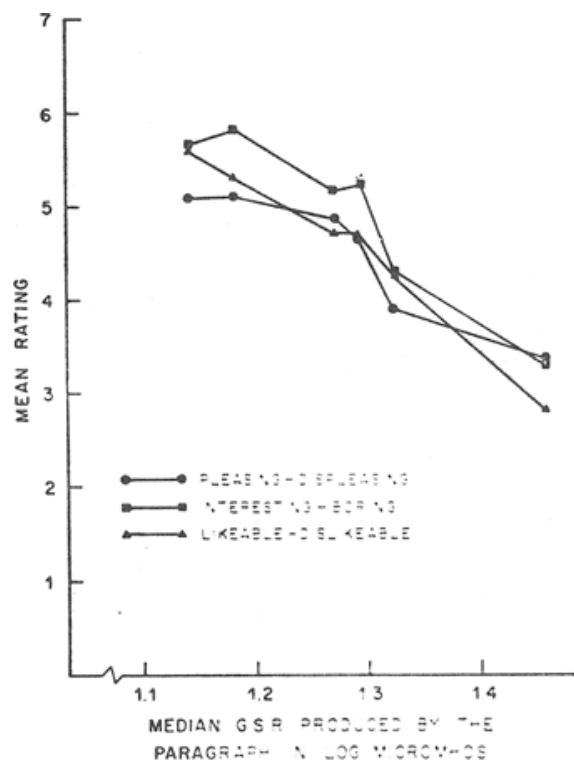

Fig. 3. Paragraph arousal level and mean ratings of pleasingnes, interestingness, and preference.

potential and arousal level.

\section{REFERENCES}

BERLYNE, D. E. Motivational problems raised by exploratory and epistemic behavior. In S. Koch (Ed.), Psychology: A study of science. Vol. 5. New York: MeGraw-Hill, 1963. Pp. 284-364.

BERLYNE, D. E. Arousal and reinforcement. In $D$. Levine (Ed.), Nebraska symposium on motivation. Lincoln, Nebr: University of Nebraska Press, 1967. Pp. 1-10.

DAY, $\mathrm{H}$. A subjective definition of complexity. Perceptual \& Motor Skills. $1967,25,583-584$

BVANS, D. R. Conceptual complexity, arousal, and epistemic behavior. Canadian Journal of Psychology, 1970a, 24, 249-260.

EVANS, D. R. Conceptual complexity, arousal, and epistemic behavior. Dissertation Abstracts International. 1970b, 31, 2303B (Abstract).

GRANDAGE, A. Orthogonal coefficients for unequal intervals. Biometrics, 1958 , 14. 287-289.

KAMMANN, R. Verbal complexity and preferences in poetry. Journal of Verbal Learning \& Verbal Behavior, 1966, 5 536-540. 